\title{
Tangence
}

\section{Ma vie n'est qu'un journal ou la dernière photo}

\section{Régine Robin}

Numéro 42, décembre 1993

Le récit de soi

URI : https://id.erudit.org/iderudit/025786ar

DOI : https://doi.org/10.7202/025786ar

Aller au sommaire du numéro

Éditeur(s)

Tangence

ISSN

0226-9554 (imprimé)

1710-0305 (numérique)

Découvrir la revue

Citer ce document

Robin, R. (1993). Ma vie n'est qu'un journal ou la dernière photo. Tangence, (42), 12-26. https://doi.org/10.7202/025786ar d'utilisation que vous pouvez consulter en ligne.

https://apropos.erudit.org/fr/usagers/politique-dutilisation/ 


\section{Ma vie n'est qu'un journal \\ ou la dernière photo}

Régine Robin

\section{Première journée}

Ciel blafard. Je me tiens tapie au fond de mon bureau. Rester toute la journée dans mes livres, ne même pas s'apercevoir s'il fait beau ou non, quand le soir descend. Rivée à mon journal, l'écriture de ceux qui sont sans écriture. La mémoire des amnésiques. Je passe mon temps à anticiper ce que je vais écrire dans mon journal, comment je vais l'écrire et à revenir en arrière. Qu'est-ce que j'ai fait en février 1988? L'angoisse! .Ai-je seulement vécu, survécu? Je feuillette l'année 1988, j'arrive au mois de février, je lis: "Je ne sais pas pourquoi je me suis lancée dans l'aventure du Kafka. Je n'ai que cent pages d'écrites, pas l'essentiel en tous les cas. Ce livre, je le portais en moi. Ce serait non pas un Kafka, mais le Kafka! Au bout de cent pages: "Ce n'est qu'un livre", je dois déchanter. J'ai du mal à avancer. Les mots ne me viennent pas. Kafka résiste. Avec Denise nous projetons d'aller à Prague cet été, juste après Berlin. Je pourrai refaire l'itinéraire habituel, aller voir sa tombe au cimetière. "Je referme le cahier. Terrassée. Denise, mon amie, nos projets, notre jeunesse. Denise vivante. J'ai mal à son absence.

Qu'est-ce qui la portait vers les canaux, vers les écluses? Pourquoi Rogny-les-Sept-Écluses? Un texte sur l'étrange mort de mon amie intime Denise, mort totalement absurde et en même temps totalement surdéterminée, complètement dans sa manière, mort mystérieuse, inexplicable. Une énigme encore pour moi.

D'abord, reconstruire par le détail cette dernière journée, la journée fatale du vendredi 28 août. Faisait-il beau? Sans doute, car Denise n'aurait pas eu l'idée de prendre des photos sans une belle lumière, douce ou vive. Il lui fallait un rayon de soleil, une ombre sur un portail, un nuage sur un champ d'orge. Il devait faire beau. Denise est chez Béatrice, dans un village du Loiret. Elle s'est arrêtée chez elle sur le chemin du retour. Bientôt Paris. Mais elle n'est pas pressée. Elles décident de partir à Bué, près de Sancerre, pour acheter du vin. Elles partent en voiture. Le plus 
jeune fils de Béatrice est aussi du voyage. En fait, Denise devait passer le week-end dans la maison familiale de Ramerupt dans l'Aube, mais tous ces villages ne sont pas très éloignés les uns des autres et cela promet de belles ballades. Après tout, on est encore en vacances. Dominique lui a donné une bonne adresse à Bué, à ne pas négliger. C'est la fin de l'été. De la douceur dans l'air. Denise sera à Paris le lundi 7 septembre même si l'Université ne reprend pas immédiatement. Elle aimait bien se faire tous les ans sa réserve de vin pour l'hiver. Quand j'arrivais de Montréal, un de nos rituels favoris était d'ouvrir la bouteille des retrouvailles qui, en général, était suivie de beaucoup d'autres - nous buvions sec toutes les deux, donnant facilement dans le fameux "c'est ce que nous avons connu de meilleur" de Flaubert - et Denise me faisait le récit de l'endroit d'où la bouteille provenait, du jour où elle était allée la chercher, généralement dans la région de Cahors, mais cette année, c'était à Sancerre qu'elle voulait aller car elle avait une bonne adresse, à Bué. Le retour à Paris par le chemin des écoliers, par Sancerre via Montbouy, village du Loiret, où Béatrice habite, avant de s'arrêter à Ramerupt. Elle venait du Mans où elle s'était arrêtée quelques jours pour voir Dominique, une autre amie qu'elle aimait beaucoup, dont elle me parlait souvent, amie qui avait depuis peu adopté une petite fille originaire du Brésil. Cette petite Alexandra s'était mise à compter énormément pour Denise qui n'avait pas eu d'enfant et qui n'arrivait pas à se remettre de ce manque, fondamental, pour elle. Le matin du 28 , elle regarde la carte routière pour repérer son itinéraire. Béatrice est intriguée, un peu en dehors du coup. Soudain, Denise tombe sur le nom d'un village: Rogny-les-SeptÉcluses. Pour rien au monde, je ne manquerai un village qui s'appelle comme ça! s'écrie t-elle. Pourtant, pour aller de Montbouy à Sancerre par Rogny-les-Sept-Écluses, il faut faire un détour. Denise n'est pas pressée, elle a l'art du détour et elle veut en rentrant à Paris avoir sa cargaison de belles photos. Qu'est-ce qui l'attirait vers les canaux, vers les écluses? La photo, c'est son péché mignon, son hobby, sa passion. Je l'ai toujours connue en train de faire des photos, de montrer des photos, de projeter d'aller faire des photos même avec des appareils automatiques, de petits appareils pas trop sophistiqués. L'année prochaine, nous projetions, mon mari, Denise et moi, de faire un grand voyage dans l'Ouest américain et ce n'était que conversations sur les livres que nous devrions lire pour le préparer, les films, des westerns principalement, à voir impérativement et, bien entendu, 
14

les types d'appareil photo qu'il faudrait emporter. Claude, mon mari, voulait à cette occasion s'acheter un nouvel appareil ou se servir de l'appareil de son père - autre mordu de la photo - et s'acheter un grand angle. Immédiatement, Denise avait également eu le projet d'acquérir ce nouvel objectif pour les paysages de l'Utah et de l'Arizona pour ne pas parler du Colorado qui nous arrachait des larmes rien qu'à l'évoquer. Nous n'arrêtions pas de parler de photo. A l'annonce de sa mort, j'ai fait le tri de celles que j'ai retrouvées. J'ai ressorti toutes celles où elle figurait. Plus nombreuses, celles qu'elle avait prises. Nous avions fait ensemble tant de voyages! Amsterdam en 1972 ou 1973, avant son accident de ski où elle s'était cassé une jambe; puis, avec Francine, les abbayes baroques d'Autriche et de Bavière et Vienne; en 1982, toujours avec Francine, Venise où nous avions déniché une merveilleuse petite chambre d' hôtel avec un accès direct sur une terrasse avec vue sur les toits de Venise! A room with a view! Prague, en 1988, alors que je terminais mon livre sur Kafka et notre programme de photos consistait à prendre tous les lieux qui avaient une signification dans la vie ou l'œuvre de Kafka: ses différents lieux d'habitation, sa petite école, le gymnasium, l'université, le bureau où il travaillait quasiment tous les jours, l'endroit où Löwy avait donné les représentations du théâtre yiddish qui avaient eu un tel retentissement sur Kafka, le Belvédère où je l'ai prise en photo à cause de ce passage du journal de Kafka, "seul au Belvédère sous les étoiles", la tombe de Kafka dans le nouveau cimetière tout au bout d'une interminable ligne de métro, le vieux cimetière juif, tout Prague en un sens. Nous étions aussi allées à Londres en 1990 alors que j'étais malade et que je me traînais avec une canne. Nous avions passé tout notre temps à la National Gallery, moi sur un fauteuil roulant qu'elle traînait. Londres par temps de canicule! Communiant, elle et moi, dans les bleus de Piero della Francesca et les MichelAnge, sans parler des Turner de la Tate Gallery où nous nous attardâmes également. Tu ne peux pas savoir l'effet que me fait la peinture! me répétait-elle souvent. Je volerais si je pouvais.

\section{Deuxième journée}

J'ai relu Parcours de E. Jabès. J'ai beaucoup aimé le verbe écrit "le v'herbe" avec le mot herbe devant le $v$ 'qui fait penser au "vav" hébreu, la lettre de la réversibilité du temps. De même que 
l'imprononçable qui s'écrit: l'in (un) pronom-sable. L'écriture. Le désert.

Le temps fuit comme un vieux stylo. Rêve curieux. Traversée d'un hall avec de grandes bibliothèques. Une scène curieuse de douane. Je me fais pincer. Je me réveille en sursaut. Aucun reste diurne en dehors des bibliothèques. Je vis dans les bibliothèques, dans ma bibliothèque. Je vis autour de mon journal. Toujours autant de mal à me dessaisir, toujours confrontée au problème de "tout garder", de l'impossible mais nécessaire castration symbolique. Vision de spectacle grotesque à la télévision. Les défenseurs du parlement russe, une "armée" improvisée en costumes de loges de théâtre, avec des drapeaux rouges ressortis on ne sait d'où, une vieille femme édentée attisant le feu d'une barricade. Spectacle, mise en scène de la désespérance; on ressort les vieux emblèmes comme des armes dérisoires. Tout est allé trop vite sans que personne n'y comprenne rien. Images en direct ou quasiment en direct. La foule entrant dans les locaux de la télévision, des balles qui sifflent, un camion qui enfonce les portes comme un bélier, des drapeaux, des cris. Tout cela noyé dans le sang, comme prévu. Révolte hybride. D'anciens communistes, des voyous, des vieilles femmes qui ont tout perdu avec leur pension, des fascistes, des paumés. Nous ne verrons pas la fin de cette gabegie de notre vivant. Quelque chose de notre monde s'est écroulé dans les années 1980-1990 et nous survivons dans des événements désormais indécodables.

En mai 1992, nous sommes parties Denise et moi pour quelques jours en Bourgogne, à ma demande. Ma psychanalyse me ramenait à mon passé et je traversais une phase qui consistait à remettre mes pas dans tous les endroits où j'étais passée. Bref, je voulais revoir la Bourgogne où j'avais été nommée professeur après l'agrégation en 1963, où j'avais rencontré Gérard, mon premier mari, que Denise connaissait, et où ma fille Anny était née. Denise acheta pour l'occasion un nouvel appareil, à la FNAC, le plus perfectionné des appareils automatiques. Elle en avait pourtant un autre que sa mère lui avait offert, qu'elle ne sortait que pour les grandes occasions, un appareil qui exigeait des mises au point compliquées, sur lequel on pouvait adapter des objectifs variés, des filtres, et qui était lourd car elle devait le traîner dans une malette spéciale. Fatiguée, pensant qu'il nous encombrerait, elle avait décidé de le laisser chez elle, mais elle avait acheté un nouvel appareil pour la circonstance. Je ne 
compte pas le nombre de fois où elle est venue à Montréal, où nous sommes allées à New York ou à Boston, toujours avec la mission de faire des photos. Je me suis mise à regarder les photos du voyage en Bourgogne. Quelques églises, moi devant mon ancien lycée, des gisants et pleurants du musée de Dijon, quelques belles maisons anciennes, des églises, des villages tassés au fond de quelque vallon, et surtout des photos par dizaines du canal de Bourgogne, des champs de moutarde sous des ciels noirs, des ponts, des berges, des écluses, des maisons d'éclusiers. Quelque chose attirait Denise au bord des écluses, le long des canaux. Qu'est-ce qui l'attirait le long des canaux, auprès des écluses? En Bourgogne, elle me parla de la beauté du canal de Briare qu'elle avait découvert en faisant une randonnée avec des amis. Elle aimait prendre les écluses en photo, trouver des angles imprévus, profiter de rayons de lumière insolite, saisir le moment, sachant qu'il suffisait d'une seconde pour que tel rayon de soleil sur les toits disparaisse, que tel sourire sur un visage s'efface. À la recherche du fugace, de l'éphémère, de l'instantané, du saisissement. S'acharner à saisir ce qui passe, ce qui meurt. Elle voulait d'autant plus prendre des photos ce jourlà, que l'endroit de Bretagne où elle avait passé le plus clair de ses vacances, à Pénestin dans le Morbihan, ne lui avait pas plu. Quelconque, sans cachet, dans un lieu trop urbanisé, colonisé par les vacanciers. Elle restait nostalgique d'une certaine maison de l'île d'Yeu où elle et de nombreux amis (je n'y suis jamais allée, il faudrait se demander pourquoi) avaient passé le mois de juillet ou le mois d'août, régulièrement pendant près de quinze ans; maison sauvage dans une région sauvage, accessible uniquement par bateau à partir de Fromentine; grande maison où l'on pouvait tout à la fois s'isoler pour lire ou pour travailler et se retrouver tous ensemble. Ils étaient parfois une vingtaine dans la maison blanche du vieux Jules Denis. Et puis, la famille a repris la maison, il a fallu trouver autre chose. Mais rien ne l'a jamais remplacée. On fit les projets les plus fous. Il était question l'an dernier que cette troupe se retrouve sur la côte dalmate en Croatie, juste au moment où la guerre faisait rage. Cette année, ils avaient trouvé cette maison du Morbihan mais elle ne plut pas du tout à Denise qui renonça à prendre la moindre photo. Elle avait écrit à son amie antiquaire à Paris: "La Bretagne est envahie et on se prend à rêver d'île déserte". En arrivant chez Béatrice, et en découvrant qu'avant de reprendre sa vie au rythme fou à Paris, elle pouvait encore voir un canal et s'arrêter à ce village au nom 
si pur: Rogny-les-Sept-Écluses, elle ne résista pas. Sept le chiffre sacré, et sept écluses à la suite, une promesse de bonheur.

\section{Troisième journée}

Je me suis réveillée avec un grand mal à New York comme on a mal à la tête. Écrasée de travail. Je ne sais quand je vais pouvoir aller à New York. Tout me manque. Greenwich et les galeries de Soho, le Manbattan Bistro et les librairies du Village, le Midtown et les boutiques et les bars, le salon de the du Plaza et les cocktails de la terrasse du Beekman Tower, le MOMA et Broadway, le Uptown et la couleur du ciel en automne. J'ai mal à l'odeur des petits pains ronds de la Bakery de la rue Bleeker. Tout me manque, les gratte-ciels et les maisons de la $72^{\mathrm{e}}$ Rue, le métro et les taxis jaunes, le New York Times du dimanche et la New York Public Station de la radio. Écrasée de travail. Trois articles à faire en même temps, un chapitre de livre et les traductions, sans compter les cours et le reste. À peine le temps de respirer. La lutte contre la montre. Je rêvasse néanmoins, j'invente des bouts d'histoire sans liens, des scénarios, des textes qui viendraient se tresser, se détresser comme remède à ma détresse; des textes comme celui-ci.

Un jour de juin, il y a quelques années, il m'est arrivé une singulière aventure. J'ai d'abord voulu garder l'histoire pour moi, n'en laissant échapper que quelques bribes sur le divan du psychanalyste, par ci, par là, où dans quelque salon à la mode pour faire mon intéressante, mais jamais personne n'a réussi à démêler l'écheveau d'une aventure inquiétante qui a failli mal tourner et surtout pas moi.

J'étais donc assise à la terrasse du Sélect comme à mon ordinaire, attendant Godot ou l'inspiration, lisant Le monde, parcourant L'officiel des spectacles, fumant mes St-Moritz menthol que je venais d'acheter au bureau de tabac du Dôme, en face, habillée de ma veste de lin que je trimballais partout en cette fin de printemps. J'allais mal, comme à mon ordinaire, enfin, un peu plus mal qu'à l'ordinaire. Mon mari ne me téléphonait pas, bien à l'abri de l'autre côté de l'Atlantique, entouré de ses nénettes, débarrassé pour quelque temps de sa femme, et mes affaires de cœur de ce côté de l'océan s'annonçaient plutôt mal, de même que mes histoires d'éditeurs. Je n'arrivais pas à venir à bout d'un 
texte qui s'intitulait: "Souvenirs de déglingue: mes insomnies" et qui était censé raconter mes déambulations solitaires dans un Paris méconnaissable livré à la spéculation immobilière, au chômage et à la galère; la radio, il ne valait mieux pas l'ouvrir. On se massacrait gaillardement un peu partout. Quant à la télévision, la télé...

Je n'avais rien dans mon frigidaire. J'avais bien fait le marché le long du boulevard Raspail à la hauteur du métro EdgarQuinet, mais c'était pour écouter le joueur d'orgue de barbarie ("Moi qui l'aimais tant, c'était le plus beau des gars de la SaintJean", un air comme ça) et acheter des coquelicots. Mon imprimante était en panne et le texte, comme on dit du courrier, en souffrance. Je n'étais pas pressée de rentrer chez moi, je traînais au Sélect, descendant bourbon sur bourbon, m'absorbant dans la lecture de la notice nécrologique du Monde, cherchant un film pour la soirée.

Il faisait beau, il faisait bleu, il faisait rose, un temps à vous couper le souffle, à faire catleya, mais pas tout seul et j'étais seule, tout à fait paumée. Je n'avais même pas envie d'appeler ma fille qui était pourtant de passage à Paris, arrivée depuis peu d'Israël et qui m'avait fait savoir qu'elle cherchait à tout quitter làbas, à partir, sans savoir où atterrir exactement. À New York, à Montréal, à Paris, allez savoir. Où émigrer, où s'installer, où peuton encore se réchauffer les miches aujourd'hui? Bref, to make a long story short, je descendais mes bourbons, je fumais mes cigarettes, levant parfois le nez, dévisageant les passants ou les autres consommateurs, tous plus jeunes que moi, d'un autre monde, rien avoir avec. Soudain, je vois s'approcher vers moi une femme entre deux âges, jolie, bien maquillée, portant un petit tailleur de soie plutôt ricos et, m'adressant la parole avec un fort accent américain, me dit:

- Ne seriez-vous pas Pamela Wilkinson par hasard?

Je ne sais pas pourquoi, j'ai répondu aussitôt sans réfléchir:

- Oui, c'est bien moi.

La dernière fois qu'au Sélect quelqu'un m'avait abordée pour me demander si j'étais machine chose ou truc muche avec des noms à coucher dehors, j'avais répondu:

- Hélas non, mais je sens que ça aurait été intéressant. 
Et puis, l'histoire s'arrête. Ciel bleu pâle. Des feuilles mortes, bientôt la pluie. Je ne sais pas pourquoi me trotte dans la tête cette phrase de Proust à propos de la pluie: "Quand par les soirs d'été le ciel harmonieux gronde comme une bête fauve et que chacun boude l'orage, c'est du côté de Méséglise que je dois de rester seul en extase à respirer à travers le bruit de la pluie qui tombe, l'odeur d'invisibles et persistants lilas."

\section{Quatrième journée}

Beaucoup de travail. J'ai attendu de mordre dans mon journal comme dans un fruit défendu. Rumination sur le corps.

À corps perdu.

Le corsage. Le corps sage. Corps sans âge.

Le corps sain. Le corset. Le corps sait.

À mon corps défendant.

Le diable au corps.

Être perdue corps et biens. Couler. Mon beau navire ô ma mémoire.

Le corps du délit.

Le corps délié. Le corps délie.

Le corps du lit.

La levée du corps.

Corps à corps.

Prendre corps.

Le corpus.

Le corps glorieux.

S'en aller du corps. Déféquer ou mourir?

Garde du corps.

Prise de corps. Le corps prisé, reprisé, épuisé.

Corps céleste.

Le corps d'une lettre.

Avoir du corps. 
Corps de lois.

Corps de garde.

Esprit de corps.

Journal mémoire. Journal fourre-tout. Tantôt un état d'âme (pas trop), tantôt des esquisses de travaux, de réflexions, de citations. Vivier, répertoire, réserve de textes de notes. Tantôt ce sont des carnets de voyage, des impressions après des séances d'analyse, tantôt des aperçus sur le travail de recherche. Des mots, des phrases, des plans, des scénarios. Decay. Ce mot me frappe. Des K. Délabrement, dissolution, l'engluement du temps et de l'Histoire.

Avant de s'arrêter chez Béatrice, Denise avait fait un crochet par Le Mans pour voir Dominique, comme ça, pour laisser couler la vie auprès de quelqu'un qu'elle aimait. Elle s'était aussi arrêtée à Nantes (il pleut sur Nantes...), avait fait le tour du Nantes du $\mathrm{xvIII}^{\mathrm{e}}$ siècle, et sans doute avait-elle pris des photos. Je suis frappée par l'art de vivre de Denise, par cette qualité si rare, si délicate, de rendre les autres meilleurs à son contact, de les illuminer. Ses vacances, moment toujours redoutable lorsqu'on vit seul, Denise les organisait. C'était en général la tournée des amis, sauf les années où elle faisait un grand voyage, comme lorsqu'elle venait chez nous, de l'autre côté de l'Atlantique, ou lorsque comme autrefois elle participait à quelque voyage organisé à Cuba ou dans l'ex-Union soviétique, qui à l'époque n'était pas ex du tout... Elle savait mêler le travail où elle était d'une exigence rare, la lecture - elle avait emporté un livre de Nabokov et un roman de Tondelli - l'amitié, le repos, les plaisirs de la bonne bouffe et le sport: la nage, la randonnée. Elle était partie de Paris le 15 juillet pour Marseille pour travailler avec Jacques à la mise au point d'un article d'analyse du discours qui devait paraître dans la revue de linguistique Langages. Elle y avait déjà beaucoup travaillé durant la première quinzaine de juillet, mais là, il s'agissait de le terminer par un travail intense en commun. Denise traversait une crise intellectuelle et cet article d'analyse du discours lui importait au plus haut point. De Marseille et de Cassis, elle est partie près de Toulon où séjournait sa mère, puis elle est revenue à Paris. Le temps de changer de valise, de laver quelques fringues, de prendre quelques livres et de donner quelques coups de téléphone, mais Paris est presque désert à la fin juillet. Elle a pris sa voiture, et elle a foncé en 
Bretagne le $1^{\text {er }}$ août. Elle ne quittera Pénestin en Morbihan que le 18 août pour Cerisy. Elle suivit la semaine de séminaire dévolue à Saussure, pris des notes, participa aux travaux et quitta Cerisy rayonnante. Le colloque lui avait donné des idées, elle y avait retrouvé des amis et noué sans doute de fructueux contacts. Au Mans, Dominique se souvient d'un moment particulièrement lumineux. Elle rentre dans la pièce où travaille Denise. C'est la fin de l'après midi. Denise se retourne. Elle est traversée par une flaque de soleil. Elle lui dit, radieuse: "Je viens de relire mes notes de Cerisy, je suis contente, j'ai bien avancé. Elle revint en Bretagne pour quelques jours et partit pour Le Mans le 25. Au Mans, Dominique avait fait agrandir deux photos que Denise avait prises, lors de notre voyage en Bourgogne, l'an dernier. Il s'agissait de pleurants du tombeau du musée de Dijon, l'un en marbre, l'autre en bois peint. Dominique avait donc chez elle des agrandissements des photos de Denise. Elles en ont plaisanté, mais gravement, parce que ces photos touchaient à la mort. Il s'agissait de tombeaux et de pleurants. Denise aimait envoyer des cartes postales et des reproductions de tableaux de peinture. Elle pestait contre la piètre qualité des cartes postales à travers le monde, sauf exception. Dans tous ses voyages, le moment où elle choisissait ses cartes postales pour les amis était un moment sacré. De même, celui qui était dévolu à leur écriture.

\section{Cinquième journée}

Souvenir violent d'une séance d'analyse en juin dernier. Pourquoi être à Montréal? Pourquoi ne pas être à Paris? Quelle place? Comment gérer l'entre-deux? Est-ce gérable? Quel vilain mot de management! Toute une chaîne d'associations à partir de cet entre-deux. Mais en gros, ça se ramène à l'argument du chaudron. Parce que je suis ici avec Claude. Si je voulais rentrer, j'aurais un poste à l'Université mais à Peteroushnok et pas à Paris, alors over my dead body. La proximité des États-Unis m'attire. Grand désir d'Amérique, de déserts. À trois quarts d'heure de New-York, la ville des villes, à trois-quarts d'heure de Boston, à quelques heures de virée en voiture au Vermont pour voir les feuilles en automne, et en été, les petits clochers de villages idylliques et puis la proximité de Cambridge et de l'Université Harvard où j'ai tant de souvenirs... J'aime l'aspect inachevé du Québec, cette fausse fragilité qu'il affiche en dépit de son poids 
de glu de mémoire. Quelque chose m'attire et me garde dans ce pays borgesien impossible, dans cette identité qui cherche à se fermer en permanence et qui, en fait, est toujours ouverte. Je persiste à continuer mon scénario, ma petite histoire qui se passe à la terrasse du Sélect, vous savez au moment où une femme inconnue m'adresse la parole.

Un sourire de part et d'autre et les choses en étaient restées là. Ce jour de juin, poussée par quelque diable, j'ai répondu "oui" sans réfléchir, ne me demandant même pas ce qu'il adviendrait de moi, si la Pamela en question qui avait manifestement rendezvous avec la belle Américaine se pointait dans les trois minutes, ne faisant pas attention à la consonance anglo-saxonne du nom et le caractère invraisemblable de mon accent français si j'avais à prononcer un seul mot en anglais. Je n'avais pensé à rien. J'avais dit "oui, c'est bien moi" comme ça par pur désœuvrement, pıre disponibilité, pour voir.

Aussitôt, la dame en question, ravie de me retrouver, de faire connaissance plus exactement, se mit à me combler d'éloges. Elle avait vu mes derniers collages, en particulier ceux que je venais d'exposer sur New York et Berlin, elle les avait beaucoup aimés, trouvé génial cette idée de faire des séries sur les villes et d'agrémenter mes collages de textes courts, énigmatiques sur des déambulations nocturnes dans les villes.

"Vous êtes un flâneur des temps modernes, au sens que Benjamin donne à ce mot. J'aime beaucoup ce que vous faites et je suis ravie que vous ayiez accepté de me rencontrer car votre secrétaire à New York, la semaine dernière, ne me donnait pas beaucoup d'espoir."

Il était encore temps pour moi de tout arrêter, de lui dire que je plaisantais, que je n'avais rien à voir avec Pamela Wilkinson, que je n'étais reliée à elle que par le fait d'écrire moi aussi, d'écrire sur les villes, de connaitre et d'aimer New York et Berlin, par le fait de tenter désespérément lors de mes déambulations dans les villes d'en saisir l'âme, ce qui foutait le camp, le petit détail qui me resterait des années après, que je n'avais jamais fait de collages, que je n'avais pas de secrétaire, que je n'étais pas à New York la semaine dernière, que je traînais au Sélect non pas comme un flâneur, mais comme une femme désespérée qui cherchait à tuer le temps dans les notices nécrologiques du Monde, les dessins de Plantu et le programme des cinémas dans l'espoir de trouver une 
bonne vieille comédie musicale américaine, un Orson Welles ou un Woody Allen. Tout ce que je risquais, c'était qu'elle le prenne mal, mais de toute façon, elle attendait Pamela Wilkinson, alors! Mais non, j'ai souri, je l'ai remerciée, fait la modeste, dit que je préparais une nouvelle exposition à Paris.

Dernière carte postale, dernière photo, dernière gravure.

Pourquoi est-ce que je ne me sens véritablement exister que par le journal? À le relire, ma vie prend corps. Le journal lui donne de l'épaisseur, de la réalité. J'existe, j'ai fixé tel ou tel élément, j'ai fait la chasse à l'éphémère, j'ai conservé quelque chose, j'ai lu, j'ai rencontré des gens, j'ai fait toutes sortes de ballades aux quatre coins du monde. Noter tous les itinéraires dans les villes. Mais le journal est toujours décevant. On attendait tant du journal de Leiris! Venir à bout de "l'énorme masse opaque". Oui, tous les jours, venir à bout de ce magma gluant et indistinct qui coule comme le sang ou comme la vie. Je me réfugie dans mon verre de vin et mes St-Moritz menthol quand les nouvelles à la télévision sont ce qu'elles sont. Ce qu'est devenue l'ex-RDA, ce que devient la Russie, et la guerre en Yougoslavie, pas à piquer de vers. Et la Slovaquie, et ce que devient l'Italie. Je n'arrive pas à y croire. Tout un siècle de luttes, d'espoirs pour en arriver là.

Ce n'est pas que le "socialisme réel" ait été de la tarte. Non. Mais quelle dérision que ces discours sur la "reconquête" de la Démocratie!

\section{Sixième journée}

Qu'est-ce qui attirait Denise vers les canaux, vers les écluses, et pourquoi ce nom lui plût-il tant que cela: Rogny-les-SeptÉcluses? En Bretagne, en effet, grâce à la présence d'une amie peintre, elles font la connaissance d'un graveur. Il vient les voir dans cette maison du Morbihan. Ses gravures tout en contraste, noires avec des effets lumineux, représentent des corps de femme avec quelque chose d'inquiétant. Celle que Denise achète en particulier joue sur ce contraste. Un buste épanoui de femme et une tête qui se défait, qui disparaît. Denise l'aime, elle y voit comme une blessure, comme une image de la fragilité, peut-être même de la mort. Au Mans, Dominique lui donne le tirage de photos d'Alexandra sa fille, photos que Denise avait réclamées 
24

lors d'un précédent séjour, le jour du 14 juillet, précisément. Achat de gravures, de tableaux, envoi de cartes postales, photos qu'on se promet, que l'on prend, que l'on fait tout aussitôt développer, qu'on s'échange, tout passe par cette médiation, cette représentation, ces repères. À Rogny, dans ce village de l'Yonne, il y a sept écluses très anciennes. On dit même qu'elles datent du temps d'Henri IV. L'ensemble est depuis longtemps désaffecté. De l'herbe et de la mousse au fond à sept mètres de profondeur. De face, l'ensemble forme comme une pyramide. Des touristes avertis s'arrêtent au village. Denise prend beaucoup de photos de face, ravie. Ils remontent tous en voiture. Béatrice a hâte d'arriver à Sancerre. Ils reprennent la route. À la sortie du village, Denise s'arrête, se gare mal. Béatrice le lui fait remarquer. Denise lui dit qu'elle n'en a que pour une seconde, le temps de prendre dans cette lumière d'après-midi, il est à peu près quatre heures, de prendre sur le côté l'enfilade des écluses, elle va trouver un coin propice, prendre comme elle le dit une dernière photo. Béatrice et son fils restés dans la voiture la laissent partir. Au bout d'une minute, à peine une minute, Béatrice, un peu agacée, dit à son fils d'aller la chercher car il faut prendre la route pour arriver à Sancerre. Le fils ne voit pas Denise ni tout près, ni au loin, ni à l'horizon. Il cherche autour de lui, soudain, il pousse un cri. Denise est au fond de l'écluse. Elle est tombée sans un cri. Le temps de retourner au village, de téléphoner, d'appeler les pompiers, le Samu, de descendre au fond de l'écluse. Tout cela est rapide et prend des siècles. Par moment, Denise ouvre les yeux. Béatrice lui parle, la réconforte mais Denise devait déjà être dans le coma, ce regard n'en est pas un, elle est déjà ailleurs, nulle part. Elle parle encore, dit, répète toujours la même phrase: "Aidez-moi à tenir ma jambe droite! Aidez-moi à tenir ma jambe droite!" Le Samu s'affaire. On met Denise dans une ambulance. Béatrice suit derrière en taxi vers l'hôpital d'Auxerre. En route, l'ambulance s'arrête par deux fois. Deux arrêts cardiaques. À l'hôpital, c'est à peine si on a le temps de lui donner des soins en dehors de massages cardiaques. Denise meurt vers 7 ou 8 heures le vendredi 28 août au soir, le jour de la Saint-Augustin. Il fait très doux, c'est encore l'été et les vacanciers regagnent Paris. Qu'estce qui poussait Denise vers les canaux, vers les écluses? Et pourquoi cette mort sans un cri? Je ne comprends toujours pas.

Je ne sais pas pourquoi nous nous réfugions - au moins quelques-uns d'entre nous - dans la littérature. La vraie vie, ces 
personnages de papier qui portent notre existence. Je ne vis que par cette manipulation du livre, manipulation physique. Vivre avec les morts. Depuis si longtemps. Correspondance effrayante de ma part avec Kafka, avec Proust, avec Perec. Étrange filiation imaginaire. Allez comprendre! Dans ce monde de l'instrumentalisation, allez savoir pourquoi on se ferait encore tuer pour "le bateau ivre".

Ma fille a adhéré à un mouvement de juifs laïcs à Paris. Quelque chose s'est donc tout de même transmis! Quelque chose qui touche à l'essentiel. Je ne sais pas comment. Tangos de Buenos Aires. La musique cadencée m'emplit la tête. Jour difficile. Temps glauque. J'ai mal au dos. Tout fiche le camp. Jour blême qui se traîne. Ce journal prend des allures d'obsession, de comptes, de bilans, d'énumérations. Comme s'il fallait fixer absolument le petit rien, le futile, l'anodin, le temps mort, une réflexion, un déplacement, un événement. L'écriture du rien qui passe, de l'éphémère, de ce qui est en train de mourir, de disparaître, de s'évanouir, de finir dans le trou noir. Je me perds, me dissipe, m'éparpille. Rien n'est prêt, tout est en chantier, tout est à reprendre. Au fait, ma petite histoire du Sélect, vous voulez savoir la suite?

"Je ne savais pas que vous parliez si bien français et sans accent, dit-elle, c'est épatant. Écoutez, je ne veux pas vous faire perdre du temps, votre secrétaire vous a sans doute mise au courant, voilà. Je reçois chez moi, à mon domicile parisien, une dizaine d'amis, des Américains, des Brésiliens, des Français, en particulier des galéristes de tout premier plan, et j'aimerais beaucoup que vous soyiez des nôtres et que vous apportiez vos dernières productions. Je vous assure qu'elles seront écoulées et à bon prix avant la fin de la soirée. Ils sont tous désireux de faire votre connaissance. Aucun ne savait que vous prépariez une exposition ici, ils seront heureux de l'apprendre. Ma réception a lieu dans quinze jours. D'après ce que votre secrétaire m'a dit, entre parenthèses, elle n'est pas très aimable, vous serez encore à Paris dans quinze jours car votre secrétaire m'a parlé d'une exposition à San Francisco le mois prochain, mais rien auparavant. C'est singulier, elle n'a pas mentionné cette exposition à Paris. Viendrez-vous? Je vous en prie."

Elle ouvrit son sac de cuir, un de ses sacs Prada qui coûtent l'équivalent de mon loyer, et en sortit une carte de visite: 
ÉMILIA MORGAN

83 AVENUE DENFERT-ROCHEREAU

75014 PARIS. TÉL. : 43391012 venir?

- Mais avec plaisir, ai-je répondu, à quelle heure faut-il

- Vers $18 \mathrm{~h} 30$.

- Très bien. Vous me voyez ravie. J'apporterai quelques-uns de mes derniers collages sur Paris. Est-ce que cela vous convient?

- Sur Paris, quelle merveille! Je me réjouis d'avance de cette soirée, répondit Émilia Morgan.

Je commençai à me sentir mal. Pamela Wilkinson, manifestement en retard, pouvait arriver d'une minute à l'autre, l'effet de mes bourbons commençait à se faire sentir, je ne savais plus quoi faire, quoi inventer, je sortis mes St-Moritz menthol et mon vieux briquet d'amadou, juste pour me donner une contenance. À ce moment, mon interlocutrice se montra très étonnée. On dit dans le monde que vous ne fumez pas, que vous êtes malade, asthmatique ai-je lu dans une interview de Time Magazine, est-ce que je me trompe?

- Pas du tout, j'ai cette interview très présente à l'esprit, mais, voyez-vous, souvent à Paris, je change quelque peu de personnalité et je me mets à fumer, je fume même beaucoup, après, dès que je rentre, je suis terrassée par une crise d'asthme et je m'arrête pour quelques semaines. Je fume du reste des cigarettes qu'on ne trouve pas en Amérique du Nord, comme vous voyez, dis-je en souriant, me souvenant de ces pauses qu'on voit chez les actrices américaines dans les films des années 50 quand elles sortent leur fume-cigarette en ambre ou en ivoire.

Mon histoire ne se termine pas. Denise ne reviendra pas. Reste le journal qui continue, qui continue, qui continue.

Octobre 1993 